\title{
Vascular smooth muscle function in type 2 diabetes mellitus: a systematic review and meta-analysis
}

\author{
David Montero • Guillaume Walther • Antonia Pérez-Martin • \\ Nestor Vicente-Salar • Enrique Roche • Agnès Vinet
}

Received: 5 March 2013 / Accepted: 5 June 2013 / Published online: 18 July 2013

(C) Springer-Verlag Berlin Heidelberg 2013

\begin{abstract}
Aims/hypothesis In type 2 diabetes, in contrast to the welldocumented endothelial dysfunction, studies assessing vascular smooth muscle (VSM) function have yielded discrepant results over the last two decades. We therefore sought to determine whether or not VSM function is impaired in individuals with type 2 diabetes.

Methods We conducted a systematic search of MEDLINE, Cochrane, Scopus and Web of Science databases, from their respective inceptions until December 2012, for articles evaluating VSM function in individuals with type 2 diabetes. A meta-analysis was performed to compare the standardised mean difference (SMD) in VSM function between individuals with type 2 diabetes and age-matched controls. Subgroup analyses and meta-regression were used to identify sources of heterogeneity.

Results Twenty-seven articles (1,042 individuals with type 2 diabetes and 601 control subjects) were included in this analysis. VSM function was significantly impaired in diabetic compared with control subjects (SMD $-0.68,95 \%$ CI $-0.84,-0.52$; $p<0.001)$. Although moderate heterogeneity among studies was found $\left(I^{2}=52 \%\right)$, no significant publication bias was detected. Subgroup analyses showed a further decline in VSM function assessed in the microcirculation compared with the
\end{abstract}

Electronic supplementary material The online version of this article (doi:10.1007/s00125-013-2974-1) contains peer-reviewed but unedited supplementary material, which is available to authorised users.

D. Montero $(\bowtie) \cdot G$. Walther $\cdot$ A. Vinet

LAPEC EA4278, Avignon University, 33 rue Louis Pasteur,

F-84000 Avignon, France

e-mail: david.montero.barril@gmail.com

D. Montero $\cdot$ N. Vicente-Salar $\cdot$ E. Roche

Applied Biology Department - Nutrition/Institute of

Bioengineering, University Miguel Hernandez, Elche, Spain

A. Pérez-Martin

Vascular Medicine Unit, Nimes University Hospital, Nimes, France macrocirculation of individuals with type 2 diabetes $(p=0.009)$. In meta-regression, VSM function in the microcirculation was inversely associated with BMI and triacylglycerols and was positively associated with HDL-cholesterol.

Conclusions/interpretation In addition to the endothelium, the VSM is a source of vascular dysfunction in type 2 diabetes. An exacerbation of VSM function in the microcirculation may be a distinctive feature in type 2 diabetes.

Keywords Meta-analysis · Type 2 diabetes mellitus .

Vascular smooth muscle function

\begin{tabular}{|c|c|}
\hline \multicolumn{2}{|c|}{ Abbreviations } \\
\hline DBP & Diastolic blood pressure \\
\hline HOMA-IR & HOMA of insulin resistance \\
\hline NMD & Nitrate-mediated dilation of brachial artery \\
\hline SAQOR & $\begin{array}{l}\text { Systematic appraisal of quality for } \\
\text { observational research }\end{array}$ \\
\hline SBP & Systolic blood pressure \\
\hline SMD & Standardised mean difference \\
\hline VSM & Vascular smooth muscle \\
\hline
\end{tabular}

\section{Introduction}

The incidence of type 2 diabetes is growing rapidly, in part because of the ageing population and sedentary lifestyle [1]. In 2010, an estimated 257 million people worldwide had type 2 diabetes [1], representing a major public health issue. Type 2 diabetes is independently related to increased risk for cardiovascular complications [2], mainly involving the accelerated development of atherosclerotic vascular changes [3].

In the study of vascular function, the aim of exogenous nitrate administration is to relax vascular smooth muscle (VSM) in an endothelium-independent manner, thus reflecting VSM function $[4,5]$. Once inside the VSM cell, 
nitrates prompt smooth muscle relaxation via a cascade of events involving the bioconversion of nitrate to nitric oxide, activation of soluble guanylate cyclase, synthesis of cyclic guanosine monophosphate and decrease in cytosolic calcium levels [6]. Endothelial and VSM dysfunction are considered to be primary signs of the early stage of atherosclerotic disease [7], with a significant prognostic role in high-risk populations $[8,9]$. Impaired endothelial function, which appears long before symptoms [10], has been consistently demonstrated in the macro- and microcirculation of individuals with type 2 diabetes (Table 1) in association with hypercholesterolaemia, hyperglycaemia, low-grade systemic inflammation and oxidative stress [11]. However, whether VSM function, usually preserved in obese individuals [12], is also impaired in those with type 2 diabetes has been debated in the last two decades, because of discrepant results in both the macro- and microcirculation (Table 1).

Given this uncertainty, we conducted a systematic review and meta-analysis of available studies comparing VSM function in individuals with type 2 diabetes and age-matched control subjects.

\section{Methods}

This review is reported according to the Meta-analysis of Observational Studies in Epidemiology (MOOSE) Group guidelines [13].

Data sources and searches Our systematic search included MEDLINE, Cochrane, Scopus and Web of Science databases, since their inceptions until December 2012. We used combinations of the subject headings: 'diabetes mellitus, type 2', 'vascular smooth muscle', 'endothelium independent', 'nitroglycerin', 'sodium nitroprusside', 'vascular function', 'vascular reactivity', 'vasodilation'. We also performed hand searching in reference citations of identified reviews and original articles selected for full-text retrieval.

Study selection To be included in this review, an observational report had to: (1) assess VSM function in individuals with type 2 diabetes; and (2) include a group of age-matched controls. In the event of multiple publications, only the most recent manuscript for a particular study population was included. Inclusion was not limited by publication status or language. Study selection was performed independently and in duplicate by two investigators (David Montero) and (Agnès Vinet). Discrepancies in inclusion/exclusion were solved by consensus or through consultation with a third reviewer (Guillaume Walther).

Data extraction and quality assessment The following variables were abstracted into a pre-formatted spreadsheet: authors, year of publication, characteristics of study participants ( $n, \%$ women, age, BMI, systolic blood pressure (SBP), diastolic blood pressure (DBP) and duration of type 2 diabetes), metabolic variables (fasting insulin, fasting glucose, $\mathrm{HbA}_{1 \mathrm{c}}$, total cholesterol, HDL-cholesterol, LDLcholesterol, triacylglycerols and NEFA) and vascular variables (region assessed, technique used, endothelial function and VSM function). The presence of concomitant cardiovascular disease and smoking was also determined. Additional$l y$, if data were unclear or were not available in the published reports, we contacted the corresponding and/or first author by e-mail to request this information. A systematic appraisal of quality for observational research (SAQOR) [14], previously applied in meta-analysis of observational studies evaluating vascular function [15], was performed to provide assessment of study quality. The SAQOR was adjusted to assess: (1) the type 2 diabetic sample; (2) the control group; (3) quality of measurement; (4) confounding variables; and (5) data. Overall, the SAQOR was scored out of 16, quality deemed better with a greater score (Table 1). Data extraction and quality assessment were performed independently and in duplicate by two investigators (David Montero) and (Agnès Vinet). Discrepancies were solved by consensus or through consultation with a third reviewer (Guillaume Walther).

Data synthesis and analysis The meta-analysis and statistical analyses were performed using Review Manager software (RevMan 5.2; Cochrane Collaboration, Oxford, UK) and Comprehensive Meta-analysis software (version 2; Biostat, Englewood, NJ, USA). The primary outcome was the standardised mean difference (SMD) in VSM function between individuals with type 2 diabetes and control subjects. SMD summary statistic allowed us to standardise macro- and microcirculation studies into a uniform scale to complete the meta-analysis. According to Cohen guidelines [16], an SMD of 0.2, 0.5 and 0.8 represents small, medium and large effect sizes, respectively. Negative SMD corresponded to impaired vascular function in individuals with type 2 diabetes compared with control subjects.

Heterogeneity between studies was assessed using $I^{2}$ statistics; $I^{2}<50 \%$ was considered to represent low heterogeneity while $I^{2} \geq 50 \%$ was considered to represent high heterogeneity. Random-effects models were used to calculate SMD when $I^{2} \geq 50 \%$ [17]. Publication bias was evaluated by estimating Begg and Mazumdar's funnel plot asymmetry and Egger's weighted regression test [18].

Potential moderating factors were explored by subgroup analyses comparing the summary results of studies grouped by sample size, age, sex, duration of type 2 diabetes, differences in variable risk factors (BMI, SBP, DBP, fasting insulin, fasting glucose, HOMA of insulin resistance (HOMA-IR), $\mathrm{HbA}_{1 \mathrm{c}}$, total cholesterol, HDL-cholesterol, LDL-cholesterol and triacylglycerols) between diabetic and control subjects, 


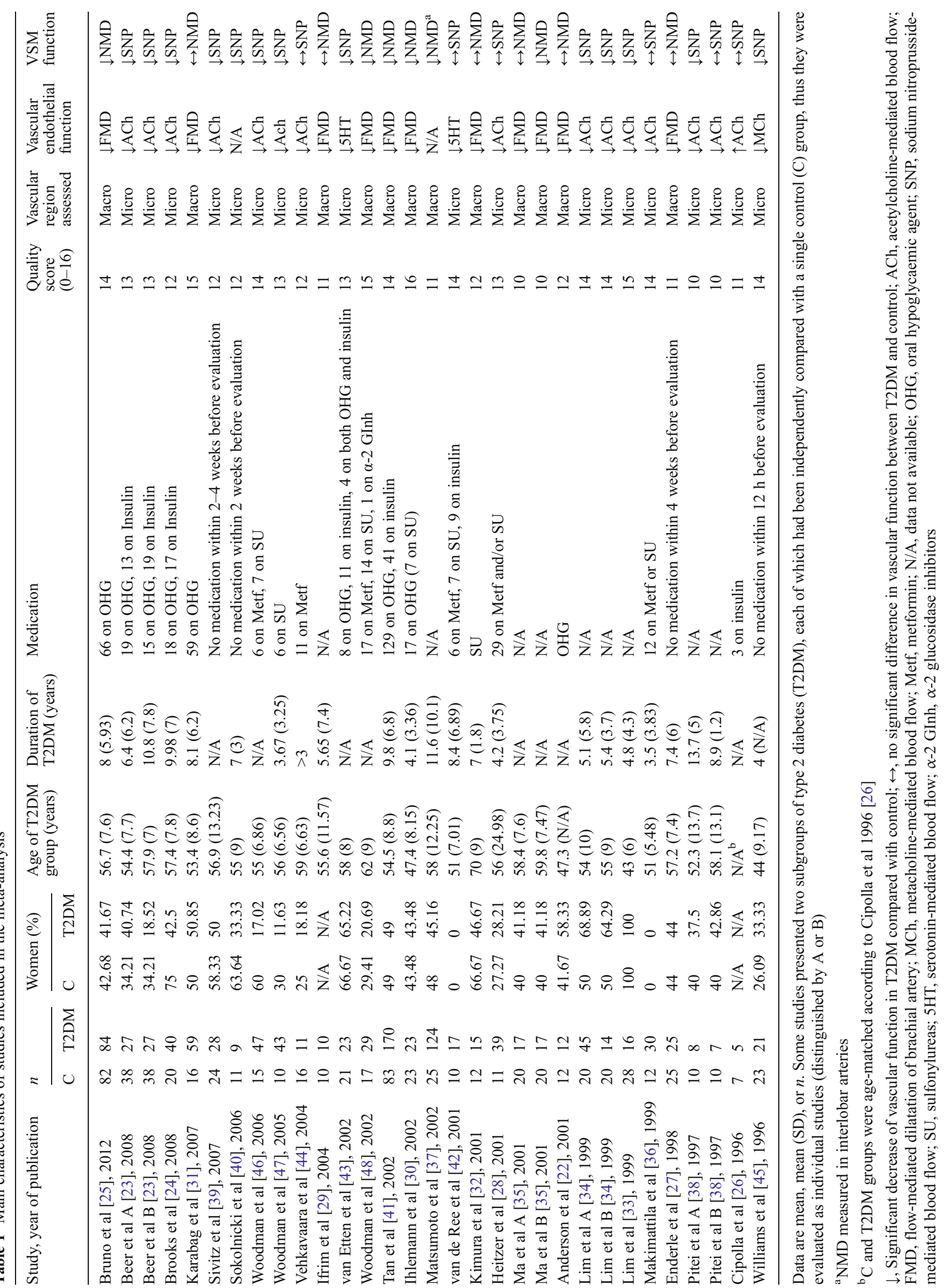


vascular region assessed, vascular technique and endothelial function. Median values of continuous variables were used as cut-off values for grouping studies. Univariate metaregression analysis was performed to further identify the possible sources of heterogeneity, using the aforementioned continuous variables.

\section{Results}

Study selection and characteristics The flow diagram of the process of study selection is shown in Fig. 1. Our search of MEDLINE, Cochrane, Scopus and Web of Science databases and manual review of articles cited in the identified and related publications initially retrieved 3,613 articles. Of these, 3,553 were excluded because they were not related to our present meta-analysis. We obtained and reviewed the full text of the remaining 60 articles, and excluded 33 for the following reasons: no VSM function data available $(n=18)$, no agematched control subjects $(n=12)$, no VSM function data reported in control subjects $(n=2)[19,20]$ or duplicate data $(n=1)$ [21]. Finally, 27 observational reports were included in the meta-analysis.

The 27 articles included in the meta-analysis encompassed 1,042 individuals with type 2 diabetes and 601 control subjects [22-48]. Four of these articles presented two subgroups of type 2 diabetes, each of which had been independently compared with a single control group, thus they were evaluated as individual studies [23, 34, 35, 38]. The characteristics of the resulting 31 studies are shown in

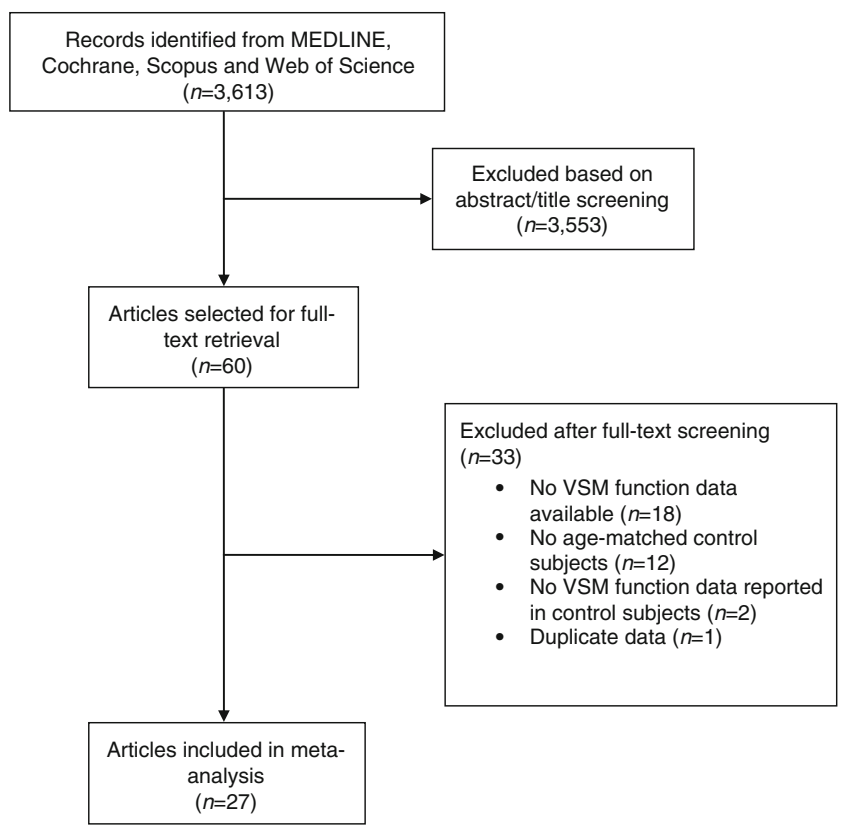

Fig. 1 Flow diagram of the process of study selection
Table 1. All of the studies compared individuals with type 2 diabetes with age-matched control subjects, ranging from 12 to 253 in total sample size. The mean clinical characteristics of all subjects in the included studies ranged from 41 to 70 years for age, 21.70 to $37 \mathrm{~kg} / \mathrm{m}^{2}$ for BMI, 112 to $156 \mathrm{mmHg}$ for SBP and 67 to $95 \mathrm{mmHg}$ for DBP. The mean fasting insulin, fasting glucose, HOMA-IR and $\mathrm{HbA}_{1 \mathrm{c}}$ ranged from 34.7 to $277.8 \mathrm{pmol} / 1$, from 4.5 to $12.6 \mathrm{mmol} / 1$, from 1.1 to 19.2 and from 4.4 to $11 \%$ (25 to $97 \mathrm{mmol} / \mathrm{mol})$, respectively. The mean total cholesterol, HDL-cholesterol, LDL-cholesterol and triacylglycerols ranged from 4.4 to $6.0 \mathrm{mmol} / \mathrm{l}$, from 0.95 to $1.78 \mathrm{mmol} / \mathrm{l}$, from 2.0 to $4.1 \mathrm{mmol} / 1$ and from 0.9 to $2.9 \mathrm{mmol} / 1$, respectively. The mean duration of type 2 diabetes ranged from 3.5 to 13.7 years. With respect to the vascular region assessed, 12 out of 31 studies evaluated the macrocirculation and 19 out of 31 evaluated the microcirculation. Impaired endothelial function was reported in 28 (11 in the macrocirculation, 17 in the microcirculation) out of 29 studies in which it was assessed and impaired VSM function was reported in 19 (6 in the macrocirculation, 13 in the microcirculation) out of 31 studies.

VSM function VSM function was determined in all of the included studies by evaluating the response to known endothelium-independent vasodilator substances related to either the macro- or the microcirculation (Table 1). The SMD in VSM function between individuals with type 2 diabetes and control subjects was used as the primary outcome. After data pooling, the meta-analysis was performed. The metaanalysis revealed that VSM function was significantly impaired in diabetic compared with control subjects (31 studies, 1,643 subjects; SMD -0.68 ; 95\% CI $-0.84,-0.52$; $p<0.001$ ) (Fig. 2). Significant heterogeneity was found in this analysis $\left(I^{2}=52 \% ; p<0.001\right)$. VSM function was also found to be significantly impaired in diabetic compared with control subjects when considering only studies assessing either the macrocirculation (12 macrovascular studies, 910 subjects; SMD $-0.46 ; 95 \%$ CI $-0.67,-0.25 ; p<0.001)$ or the microcirculation (19 microvascular studies, 733 subjects; SMD $-0.85 ; 95 \%$ CI $-1.06,-0.65 ; p<0.001)$.

Subgroup and meta-regression analyses Because we found some evidence of study heterogeneity according to potential moderating factors of each study, subgroup analyses were conducted to determine the sources of heterogeneity (Table 2). Heterogeneity was reduced below $50 \%$ of $I^{2}$ in both subgroups after dividing the studies by the difference $(\Delta)$ between diabetic and control subjects in DBP, fasting insulin, HDL-cholesterol, triacylglycerols, vascular region assessed and microvascular technique. In the remaining subgroup analyses, except for the $\Delta$ between diabetic and control subjects in $\mathrm{HbA}_{1 \mathrm{c}}$, heterogeneity was also reduced below $50 \%$ of $I^{2}$ in one of the complementary subgroups. The SMD 
Fig. 2 Forest plot of SMD in VSM function between diabetic and control subjects. IV, inverse variance; T2DM, type 2 diabetes mellitus. Squares represent the SMD in VSM function for each study. The diamond represents the pooled SMD in VSM function. Some studies presented two subgroups of type 2 diabetes (T2DM), each of which had been independently compared with a single control group, thus they were evaluated as individual studies (distinguished by A or B)

\begin{tabular}{|c|c|c|c|c|c|c|c|c|c|c|}
\hline Authors [ref.] & \multicolumn{2}{|c|}{ T2DM } & \multicolumn{4}{|c|}{ Control } & \multicolumn{2}{|c|}{$\begin{array}{l}\text { SMD } \\
\text { Weight IV, Random [95\% Cl] }\end{array}$} & \multicolumn{2}{|c|}{$\begin{array}{c}\text { SMD } \\
\text { IV, Random }[95 \% \mathrm{CI}]\end{array}$} \\
\hline Bruno et al [25] & 6.11 & 2.92 & 84 & 7.93 & 4.09 & 82 & $5.5 \%$ & $-0.51[-0.82,0.20]$ & $\rightarrow$ & \\
\hline Beer et al A [23] & 274 & 86 & 27 & 326 & 80 & 38 & $4.1 \%$ & $-0.62[-1.13,-0.12]$ & $\rightarrow$ & \\
\hline Beer et al B [23] & 255 & 65 & 27 & 326 & 80 & 38 & $4.0 \%$ & $-0.95[-1.47,-0.42]$ & $\rightarrow$ & \\
\hline Brooks et al [24] & 4.7 & 2.13 & 40 & 7.3 & 2.1 & 20 & $3.6 \%$ & $-1.21[-1.79,-0.63]$ & & \\
\hline Karabag et al [31] & 13.5 & 6.3 & 59 & 12.7 & 9.6 & 16 & $3.8 \%$ & $0.11[-0.44,0.66]$ & & \\
\hline Sivitz et al [39] & 219.54 & 88.36 & 28 & 373.44 & 208.3 & 24 & $3.6 \%$ & $-0.97[-1.55,-0.40]$ & 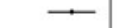 & \\
\hline Sokolnicki et al [40] & 1.91 & 0.36 & 9 & 2.38 & 0.43 & 11 & $2.0 \%$ & $-1.12[-2.09,-0.16]$ & & \\
\hline Woodman et al [46] & 9.1 & 2.74 & 47 & 13.7 & 4.26 & 15 & $3.3 \%$ & $-1.44[-2.07,-0.80]$ & & \\
\hline Woodman et al [47] & 8.8 & 2.62 & 43 & 11.9 & 2.53 & 10 & $2.9 \%$ & $-1.17[-1.90,-0.45]$ & & \\
\hline Vehkavaara et al [44] & 10.7 & 2.98 & 11 & 12.6 & 4 & 16 & $2.6 \%$ & $-0.51[-1.29,0.27]$ & & \\
\hline Ifrim et al [29] & 15.4 & 6.36 & 10 & 15.7 & 8.16 & 10 & $2.3 \%$ & $-0.04[-0.92,0.84]$ & & \\
\hline van Etten et al [43] & 275 & 146 & 23 & 391 & 203 & 21 & $3.5 \%$ & $-0.65[-1.26,-0.04]$ & & \\
\hline Woodman et al [48] & 11.4 & 4.8 & 29 & 15.4 & 7.1 & 17 & $3.4 \%$ & $-0.68[-1.30,-0.07]$ & & \\
\hline Tan et al [41] & 13.2 & 4.6 & 170 & 16.4 & 5.5 & 83 & $5.8 \%$ & $-0.65[-0.92,-0.38]$ & $\rightarrow$ & \\
\hline Ihlemann et al [30] & 15.9 & 2.88 & 23 & 18.5 & 4.32 & 23 & $3.5 \%$ & $-0.70[-1.29,-0.10]$ & & \\
\hline Matsumoto et al [37] & 29.4 & 35.63 & 124 & 49.8 & 52.5 & 25 & $4.6 \%$ & $-0.52[-0.96,-0.09]$ & & \\
\hline van de Ree et al [42] & 292 & 169.05 & 17 & 348 & 133.13 & 10 & $2.6 \%$ & $-0.35[-1.13,0.44]$ & & \\
\hline Kimura et al [32] & 12.2 & 4.6 & 15 & 12.1 & 6.4 & 12 & $2.7 \%$ & $0.02[-0.74,0.78]$ & & \\
\hline Heitzer et al [28] & 13.4 & 8.12 & 39 & 14.3 & 4.31 & 11 & $3.1 \%$ & $-0.12[-0.79,0.55]$ & & \\
\hline $\mathrm{Ma}$ et al A [35] & 20.3 & 5.4 & 17 & 23.5 & 6.8 & 20 & $3.2 \%$ & $-0.51[-1.16,0.15]$ & & \\
\hline Ma et al B [35] & 14.1 & 4.6 & 17 & 23.5 & 6.8 & 20 & $2.8 \%$ & $-1.56[-2.31,-0.81]$ & & \\
\hline Anderson et al [22] & 11.1 & 2.42 & 12 & 11.5 & 3.81 & 12 & $2.5 \%$ & $-0.12[-0.92,0.68]$ & & \\
\hline Lim et al A [34] & 73 & 35 & 45 & 122 & 41 & 20 & $3.7 \%$ & $-1.31[-1.89,-0.74]$ & & \\
\hline Lim et al B [34] & 73 & 28 & 14 & 122 & 41 & 20 & $2.7 \%$ & $-1.32[-2.08,-0.56]$ & & \\
\hline Lim et al [33] & 88 & 26 & 16 & 126 & 56 & 28 & $3.3 \%$ & $-0.79[-1.42,-0.15]$ & & \\
\hline Makimattila et al [36] & 9.1 & 3.29 & 30 & 9.9 & 4.5 & 12 & $3.1 \%$ & $-0.21[-0.89,0.46]$ & & \\
\hline Enderle et al [27] & 14.3 & 9.4 & 25 & 14.9 & 8.5 & 25 & $3.8 \%$ & $-0.07[-0.62,0.49]$ & & \\
\hline Pitei et al A [38] & 2.1 & 2 & 8 & 7.02 & 2.05 & 10 & $1.3 \%$ & $-2.31[-3.57,-1.05]$ & & \\
\hline Pitei et al B [38] & 6.42 & 1.56 & 7 & 7.02 & 2.05 & 10 & $1.9 \%$ & $-0.30[-1.28,0.67]$ & & \\
\hline Cipolla et al [26] & 0.04 & 0.02 & 5 & 0.04 & 0.03 & 7 & $1.5 \%$ & $-0.00[-1.15,1.15]$ & & \\
\hline Williams et al [45] & 4.6 & 2.75 & 21 & 8.4 & 4.8 & 23 & $3.4 \%$ & $-0.94[-1.57,-0.32]$ & $\rightarrow$ & \\
\hline Total $(95 \% \mathrm{Cl})$ & & & 1,042 & & & 689 & $100.0 \%$ & $-0.68[-0.84,-0.52]$ & 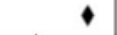 & \\
\hline \multicolumn{7}{|c|}{ Heterogeneity: $\tau^{2}=0.10 ; \chi^{2}=62.02, \mathrm{df}=30(p=0.0005) ; R=52 \%$} & & & & \\
\hline \multicolumn{7}{|c|}{ Test for overall effect; $Z=8.28(p<0.00001)$} & & & & \\
\hline
\end{tabular}

in VSM function between diabetic and control subjects was further reduced in subgroups with $\Delta$ between diabetic and control subjects in SBP $>11.00 \mathrm{mmHg}(p=0.007)$, in DBP $>3.00 \mathrm{mmHg}(p=0.007)$ and in HDL-cholesterol $\leq-0.21 \mathrm{mmol} / 1 \quad(p=0.03)$ compared with their complementary subgroups. Moreover, the SMD in VSM function between diabetic and control subjects was decreased in studies assessing the microcirculation compared with macrovascular studies $(p=0.009)$. When considering only macrovascular studies, the SMD in VSM function between diabetic and control subjects did not reach significance between any of the subgroup analyses (see electronic supplementary materials [ESM] Table 1). In microvascular studies, the SMD in VSM function between diabetic and control subjects was significantly reduced in subgroups with $\Delta$ between diabetic and control subjects in LDL-cholesterol $>0 \mathrm{~mol} / \mathrm{l}(p=0.04)$ and in triacylglycerols $>1.02 \mathrm{mmol} / \mathrm{l}(p=0.02)$, compared with their complementary subgroups (ESM Table 2). As regards the technique used for VSM function assessment, there was no significant difference between the two main microvascular techniques (iontophoresis vs plethysmography, $p=0.17$ ) (Table 2) or between the main macrovascular technique (brachial nitrate-mediated dilation [NMD]) and plethysmography $(p=0.15)$. In contrast, a significant difference was found between NMD and iontophoresis $(p=0.003)$.

Meta-regression analysis was also used to evaluate the relationship between the SMD in VSM function between diabetic and control subjects and study moderating factors. The results of this analysis are shown in Fig. 3. The $\Delta$ between diabetic and control subjects in BMI $(B=-0.04, p=0.02)$, in SBP $(B=-0.02, p=0.004)$, in DBP $(B=-0.03, p=0.003)$, in HDL-cholesterol $(B=1.19$, $p<0.001)$, in triacylglycerols $(B=-0.40, p=0.01)$ and SMD of endothelial function $(B=0.36, p=0.02)$, were significantly associated with SMD in VSM function between diabetic and control subjects. No significant association between moderating factors and SMD in VSM function between diabetic and control subjects was detected when considering only macrovascular studies. In microvascular studies, the $\Delta$ between diabetic and control subjects in BMI $(B=-0.06, p=0.048)$, in HDL-cholesterol $(B=1.03, p=0.003)$ and in triacylglycerols $(B=-0.50, p=0.03)$, were significantly associated with the SMD in VSM function between diabetic and control subjects.

Quality assessment and potential bias Based on the quality assessment criteria, 23 out of 31 studies included in the metaanalysis (quality score $\geq 12$ ) were considered to have a lowbias risk, and the remaining eight studies were considered to be of moderate-bias risk (quality score of 10 or 11) (Tables 1 and 3). The funnel plot for SMD in VSM function of studies included in the meta-analysis was notably symmetrical, suggesting the absence of a significant publication bias (Fig. 4). Also, no significant funnel plot asymmetry was detected by Begg and Mazumdar's rank correlation test $(p=0.97)$ or Egger's test $(p=0.52)$. 
Table 2 Subgroup analyses of the SMD in VSM function between subjects with type 2 diabetes and control subjects

\begin{tabular}{|c|c|c|c|c|c|}
\hline \multirow[b]{2}{*}{ Group } & \multicolumn{2}{|l|}{ Studies } & \multicolumn{3}{|l|}{ VSM function } \\
\hline & Number $^{\mathrm{a}}$ & References & $\operatorname{SMD}(95 \% \mathrm{CI})$ & $I_{\text {Heterogeneity }}^{2}$ & $p_{\text {Difference }}$ \\
\hline \multicolumn{6}{|l|}{$n$} \\
\hline$\leq 44$ & 16 & {$[22,26,29,32-36,38,40,42-45]$} & $-0.64(-0.91,-0.38)$ & 46 & 0.75 \\
\hline$>44$ & 15 & {$[23-25,27,28,30,31,34,37,39,41,46-48]$} & $-0.68(-0.90,-0.49)$ & 59 & \\
\hline \multicolumn{6}{|l|}{ Mean age } \\
\hline$\leq 54.90$ years & 15 & {$[22,29-31,33,34,36,38,40-42,45,46]$} & $-0.72(-1.00,-0.45)$ & 61 & 0.70 \\
\hline$>54.90$ years & 15 & {$[23-25,27,28,32,35,37,39,43,44,47,48]$} & $-0.66(-0.85,-0.46)$ & 43 & \\
\hline \multicolumn{6}{|l|}{ Sex } \\
\hline$\leq 41.67 \%$ women & 14 & {$[23,28,35,36,38,42,44-48]$} & $-0.79(-1.05,-0.53)$ & 50 & 0.38 \\
\hline$>41.67 \%$ women & 15 & {$[22,24,25,27,30-34,37,39-41,43]$} & $-0.64(-0.85,-0.43)$ & 55 & \\
\hline \multicolumn{6}{|l|}{ Mean duration of $\mathrm{T} 2 \mathrm{DM}^{\mathrm{b}}$} \\
\hline$\leq 7$ years & 12 & {$[23,28-30,32-34,36,40,45,47]$} & $-0.70(-0.97,-0.43)$ & 46 & 0.54 \\
\hline$>7$ years & 10 & {$[23-25,27,31,37,38,41,42]$} & $-0.58(-0.85,-0.31)$ & 63 & \\
\hline \multicolumn{6}{|l|}{ Difference in BMI } \\
\hline$\leq 3.05 \mathrm{~kg} / \mathrm{m}^{2}$ & 12 & {$[28-31,35-37,40,41,43,48]$} & $-0.54(-0.76,-0.32)$ & 42 & 0.07 \\
\hline$>3.05 \mathrm{~kg} / \mathrm{m}^{2}$ & 14 & {$[22-25,27,33,34,39,42,45-47]$} & $-0.83(-1.06,-0.61)$ & 52 & \\
\hline \multicolumn{6}{|l|}{ Difference in SBP } \\
\hline$\leq 11.00 \mathrm{mmHg}$ & 11 & {$[27,30,33,35-37,41-43,47,48]$} & $-0.58(-0.74,-0.42)$ & 0 & 0.007 \\
\hline$>11.00 \mathrm{mmHg}$ & 8 & {$[23-25,34,35,46]$} & $-1.05(-1.35,-0.74)$ & 59 & \\
\hline \multicolumn{6}{|l|}{ Difference in DBP } \\
\hline$\leq 3.00 \mathrm{mmHg}$ & 10 & {$[24,25,27,30,35,37,41-43,48]$} & $-0.59(-0.73,-0.44)$ & 0 & 0.007 \\
\hline$>3.00 \mathrm{mmHg}$ & 9 & {$[23,33-36,46,47]$} & $-1.02(-1.29,-0.74)$ & 42 & \\
\hline \multicolumn{6}{|l|}{ Difference in fasting insulin } \\
\hline$\leq 79.17 \mathrm{pmol} / \mathrm{l}$ & 6 & {$[33,36,44,46-48]$} & $-0.81(-1.17,-0.45)$ & 41 & 0.44 \\
\hline$>79.17 \mathrm{pmol} / 1$ & 6 & {$[23,34,39,45]$} & $-0.98(-1.21,-0.74)$ & 0 & \\
\hline \multicolumn{6}{|l|}{ Difference in fasting glucose } \\
\hline$\leq 3.80 \mathrm{mmol} / 1$ & 12 & {$[23,25,27,34,36,41-43,46-48]$} & $-0.70(-0.91,-0.49)$ & 48 & 0.96 \\
\hline$>3.80 \mathrm{mmol} / 1$ & 11 & {$[22,28,31,33-35,39,45]$} & $-0.69(-1.01,-0.37)$ & 57 & \\
\hline \multicolumn{6}{|l|}{ Difference in HOMA-IR } \\
\hline$\leq 5.26$ & 6 & {$[23,34,36,46-48]$} & $-0.90(-1.27,-0.53)$ & 54 & 0.94 \\
\hline$>5.26$ & 6 & {$[23,33,34,39,44,45]$} & $-0.92(-1.18,-0.66)$ & 0 & \\
\hline \multicolumn{6}{|l|}{ Difference in $\mathrm{HbA}_{1 \mathrm{c}}$} \\
\hline$\leq 2.50 \%(\leq 4 \mathrm{mmol} / \mathrm{mol})$ & 11 & {$[25,28,31,33,34,36,39-41,46,47]$} & $-0.71(-0.98,-0.44)$ & 63 & 0.84 \\
\hline$>2.50 \%(>4 \mathrm{mmol} / \mathrm{mol})$ & 9 & {$[27,30,32,34,38,43-45]$} & $-0.67(-1.03,-0.30)$ & 56 & \\
\hline \multicolumn{6}{|c|}{ Difference in total cholesterol } \\
\hline$\leq 0.20 \mathrm{mmol} / 1$ & 12 & {$[22,25,27,28,30,35,36,40,41,47,48]$} & $-0.58(-0.80,-0.37)$ & 41 & 0.47 \\
\hline$>0.20 \mathrm{mmol} / 1$ & 9 & {$[31-34,42,43,45,46]$} & $-0.74(-1.13,-0.36)$ & 68 & \\
\hline \multicolumn{6}{|c|}{ Difference in HDL-cholesterol } \\
\hline$\leq-0.21 \mathrm{mmol} / 1$ & 12 & {$[22,23,25,30,34,35,39,43,44,46,47]$} & $-0.86(-1.09,-0.63)$ & 45 & 0.03 \\
\hline$>-0.21 \mathrm{mmol} / 1$ & 11 & {$[28,31-36,41,42,45,48]$} & $-0.50(-0.74,-0.26)$ & 42 & \\
\hline \multicolumn{6}{|c|}{ Difference in LDL-cholesterol } \\
\hline$\leq 0.00 \mathrm{mmol} / 1$ & 12 & {$[23,25,28,30,35,36,39,42,47,48]$} & $-0.68(-0.88,-0.48)$ & 30 & 0.85 \\
\hline$>0.00 \mathrm{mmol} / \mathrm{l}$ & 10 & {$[22,31-34,41,43,45,46]$} & $-0.71(-1.03,-0.40)$ & 66 & \\
\hline \multicolumn{6}{|c|}{ Difference in triacylglycerols } \\
\hline$\leq 0.92 \mathrm{mmol} / 1$ & 11 & {$[25,28,31,32,35,40,43-45,48]$} & $-0.55(-0.82,-0.29)$ & 49 & 0.10 \\
\hline$>0.92 \mathrm{mmol} / 1$ & 11 & {$[23,29,33,34,36,39,42,46,47]$} & $-0.86(-1.12,-0.61)$ & 42 & \\
\hline \multicolumn{6}{|c|}{ Difference in baseline brachial diameter } \\
\hline$\leq 0.10 \mathrm{~mm}$ & 6 & {$[22,27,30,31,35,41]$} & $-0.36(-0.66,-0.07)$ & 46 & 0.45 \\
\hline
\end{tabular}


Table 2 (continued)

\begin{tabular}{|c|c|c|c|c|c|}
\hline \multirow[b]{2}{*}{ Group } & \multicolumn{2}{|l|}{ Studies } & \multicolumn{3}{|l|}{ VSM function } \\
\hline & Number $^{\mathrm{a}}$ & References & $\operatorname{SMD}(95 \% \mathrm{CI})$ & $I_{\text {Heterogeneity }}^{2}$ & $p_{\text {Difference }}$ \\
\hline$>0.10 \mathrm{~mm}$ & 3 & {$[25,32,35]$} & $-0.67(-1.41,0.07)$ & 78 & \\
\hline \multicolumn{6}{|c|}{ Vascular region assessed } \\
\hline Macrocirculation & 12 & {$[22,25,27,29-32,35,37,41,48]$} & $-0.46(-0.67,-0.25)$ & 46 & 0.009 \\
\hline Microcirculation & 19 & {$[23,24,26,28,33,34,36,38-40,42-47]$} & $-0.85(-1.06,-0.65)$ & 40 & \\
\hline \multicolumn{6}{|c|}{ Microvascular technique } \\
\hline Iontophoresis & 8 & {$[23,24,33,34,38]$} & $-1.02(-1.31,-0.73)$ & 36 & 0.17 \\
\hline Plethysmography & 9 & {$[28,36,39,42-47]$} & $-0.72(-1.02,-0.43)$ & 43 & \\
\hline \multicolumn{6}{|c|}{ Vascular endothelial function } \\
\hline$\leq-0.89 \mathrm{SMD}$ & 13 & {$[22,25,28,32,33,35,36,41-43,46,47]$} & $-0.62(-0.85,-0.40)$ & 49 & 0.71 \\
\hline$>-0.89 \mathrm{SMD}$ & 12 & {$[23,24,27,29-31,34,44,45,48]$} & $-0.69(-0.97,-0.41)$ & 59 & \\
\hline
\end{tabular}

Median values of continuous variables were used as cut-off values for grouping studies. Difference of each variable risk factor was calculated as type 2 diabetes mellitus (T2DM) group value minus control group value

${ }^{\text {a }}$ Certain enrolled studies were not included because the value used for subgroup analysis was not reported therein

${ }^{\mathrm{b}}$ Mean time (years) since diagnosis of T2DM

\section{Discussion}

In this systematic review and meta-analysis, we pooled and analysed data from 31 studies comparing VSM function in subjects with type 2 diabetes and age-matched controls. The results of our analysis revealed a moderate-to-large impairment of VSM function in diabetic subjects (SMD $-0.68 ; 95 \%$ CI $-0.84,-0.52$ ). We also performed subgroup and metaregression analyses because of significant heterogeneity.
Subgroup analysis showed that there was a stronger decrease in VSM function in diabetic subjects in microcirculation studies compared with macrocirculation studies. Furthermore, VSM function was negatively associated with BMI, SBP, DBP and triacylglycerols and was positively associated with HDL-cholesterol and endothelial function (Fig. 3).

In type 2 diabetes, in contrast to the well-documented endothelial dysfunction, studies assessing the status of VSM function have provided controversial findings a

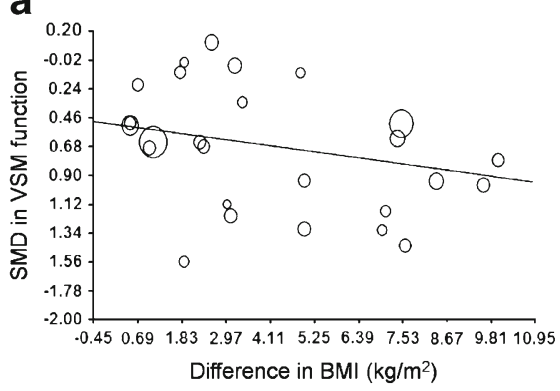

d

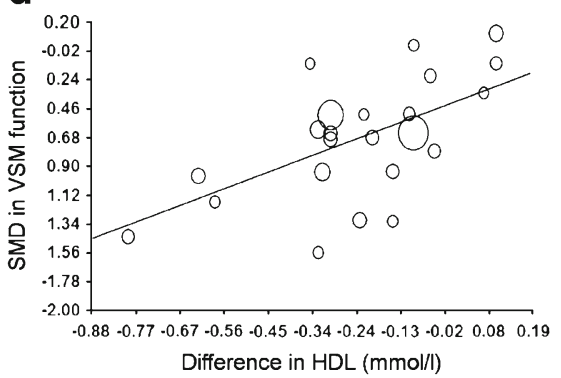

Fig. 3 Meta-regression plots of SMD in VSM function according to the difference in BMI $(B=-0.04, p=0.02)(\mathbf{a})$, in SBP $(B=-0.02, p=0.004)$ (b), in DBP $(B=-0.03, p=0.003)(\mathbf{c})$, in HDL-cholesterol $(B=1.19$, $p<0.001)(\mathbf{d})$, in triacylglycerols $(B=-0.40, p=0.01)(\mathbf{e})$ and the SMD
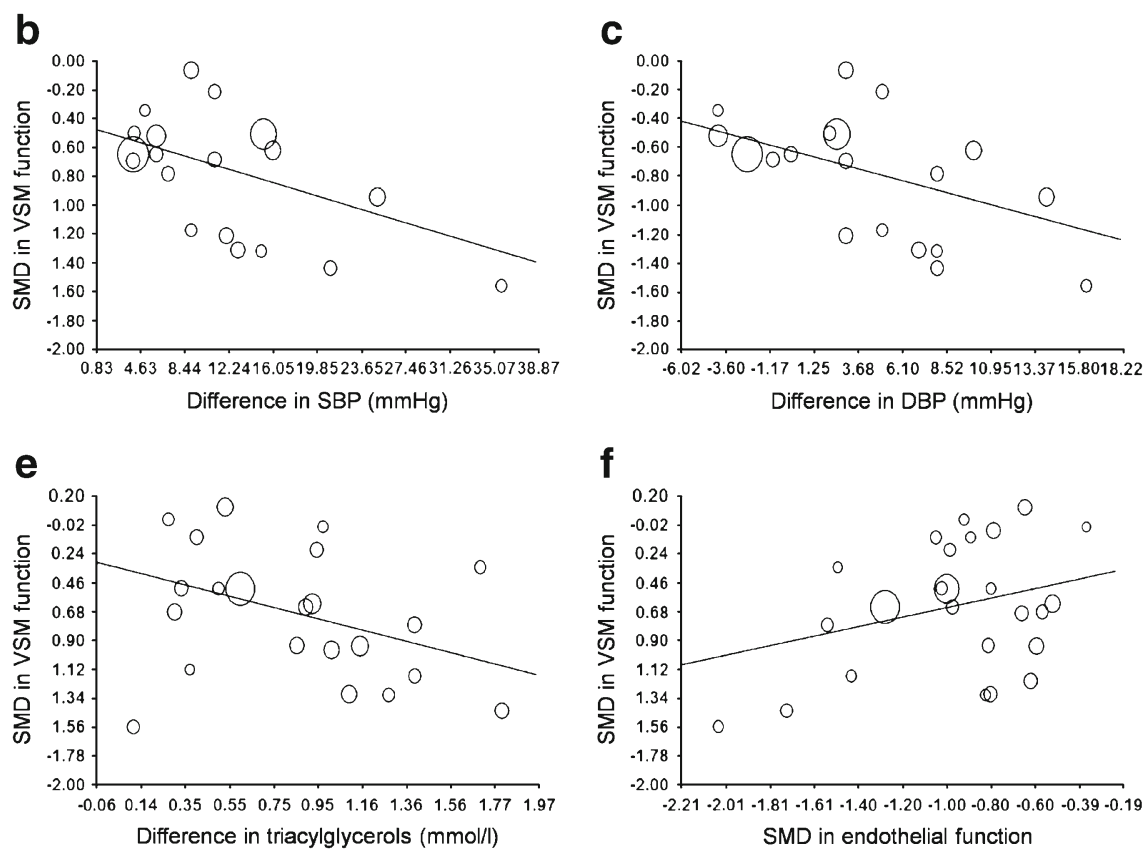

in endothelial function $(B=0.36, p=0.02)$ (f). The size of each circle is proportional to the study's weight. The difference in each variable risk factor was calculated as type 2 diabetes mellitus group value minus control group value 
Table 3 Quality assessment of studies included in the meta-analysis ${ }^{\mathrm{a}}$

\begin{tabular}{|c|c|c|c|c|c|c|}
\hline Study, year of publication & $\begin{array}{l}\text { T2DM group } \\
(0-5)\end{array}$ & $\begin{array}{l}\text { Control group } \\
(0-5)\end{array}$ & $\begin{array}{l}\text { Quality of measurement } \\
(0,1)\end{array}$ & $\begin{array}{l}\text { Confounding } \\
\text { variables }(0-3)\end{array}$ & Data $(0-2)$ & $\begin{array}{l}\text { Total quality } \\
\text { score }\end{array}$ \\
\hline Bruno et al [25], 2012 & 5 & 5 & 1 & 2 & 1 & 14 \\
\hline Beer et al A [23], 2008 & 4 & 5 & 1 & 2 & 1 & 13 \\
\hline Beer et al B [23], 2008 & 4 & 5 & 1 & 2 & 1 & 13 \\
\hline Brooks et al [24], 2008 & 5 & 4 & 1 & 1 & 1 & 12 \\
\hline Karabag et al [31], 2007 & 5 & 5 & 1 & 3 & 1 & 15 \\
\hline Sivitz et al [39], 2007 & 4 & 4 & 1 & 2 & 1 & 12 \\
\hline Sokolnicki et al [40], 2006 & 3 & 5 & 1 & 2 & 1 & 12 \\
\hline Woodman et al [46], 2006 & 5 & 5 & 1 & 2 & 1 & 14 \\
\hline Woodman et al [47], 2005 & 4 & 5 & 1 & 2 & 1 & 13 \\
\hline Vehkavaara et al [44], 2004 & 4 & 4 & 1 & 2 & 1 & 12 \\
\hline Ifrim et al [29], 2004 & 4 & 3 & 1 & 2 & 1 & 11 \\
\hline van Etten et al [43], 2002 & 4 & 4 & 1 & 2 & 2 & 13 \\
\hline Woodman et al [48], 2002 & 5 & 5 & 1 & 3 & 1 & 15 \\
\hline Tan et al [41], 2002 & 5 & 5 & 1 & 2 & 1 & 14 \\
\hline Ihlemann et al [30], 2002 & 5 & 5 & 1 & 3 & 2 & 16 \\
\hline Matsumoto et al [37], 2002 & 4 & 4 & 0 & 2 & 1 & 11 \\
\hline van de Ree et al [42], 2001 & 4 & 5 & 1 & 3 & 1 & 14 \\
\hline Kimura et al [32], 2001 & 4 & 4 & 1 & 2 & 1 & 12 \\
\hline Heitzer et al [28], 2001 & 5 & 4 & 1 & 2 & 1 & 13 \\
\hline Ma et al A [35], 2001 & 3 & 3 & 1 & 2 & 1 & 10 \\
\hline Ma et al B [35], 2001 & 3 & 3 & 1 & 2 & 1 & 10 \\
\hline Anderson et al [22], 2001 & 3 & 4 & 1 & 3 & 1 & 12 \\
\hline Lim et al A [34], 1999 & 5 & 4 & 1 & 3 & 1 & 14 \\
\hline Lim et al B [34], 1999 & 5 & 4 & 1 & 3 & 1 & 14 \\
\hline Lim et al [33], 1999 & 5 & 5 & 1 & 3 & 1 & 15 \\
\hline Makimattila et al [36], 1999 & 5 & 4 & 1 & 3 & 1 & 14 \\
\hline Enderle et al [27], 1998 & 4 & 5 & 0 & 1 & 1 & 11 \\
\hline Pitei et al A [38], 1997 & 3 & 3 & 1 & 2 & 1 & 10 \\
\hline Pitei et al B [38], 1997 & 3 & 3 & 1 & 2 & 1 & 10 \\
\hline Cipolla et al [26], 1996 & 3 & 3 & 1 & 2 & 2 & 11 \\
\hline Williams et al [45], 1996 & 5 & 5 & 1 & 2 & 1 & 14 \\
\hline
\end{tabular}

Some studies presented two subgroups of type 2 diabetes (T2DM), each of which had been independently compared with a single control group, thus they were evaluated as individual studies (distinguished by A or B)

${ }^{\text {a }}$ Adapted from the SAQOR [14]

(Table 1). Previous speculations on these inconsistent results, such as sample size and duration of type 2 diabetes, did not account for the heterogeneity in VSM function when quantitatively assessed in the meta-analysis (Table 2). The heterogeneity in VSM function could, however, be explained in part by differences in BMI, arterial pressure, and dyslipidaemia among diabetic and control subjects in the aforementioned studies (Fig. 3). The main finding of this meta-analysis was, therefore, to confirm the impairment of VSM function, in parallel with endothelial dysfunction, in type 2 diabetes. It is interesting to note that endothelial dysfunction in persons with type 2 diabetes should then be asserted with caution, given that endothelial-dependent vasodilatation depends, at least in part, on VSM function. This study suggested a novel mechanism of cardiovascular disease in persons with type 2 diabetes. VSM dysfunction was previously reported in asymptomatic adults with risk factors for atherosclerosis [49], and it was clearly demonstrated to predict future cardiovascular events even better than endothelial function in such individuals [9, 50]. Moreover, the association of VSM function with cardiovascular risk factors differed from that of endothelial function, and was more strongly related to vascular disease than endothelial function in individuals with type 2 diabetes [51]. Consequently, the prognostic significance of VSM function may be worth reconsidering in order to improve the accuracy of risk 


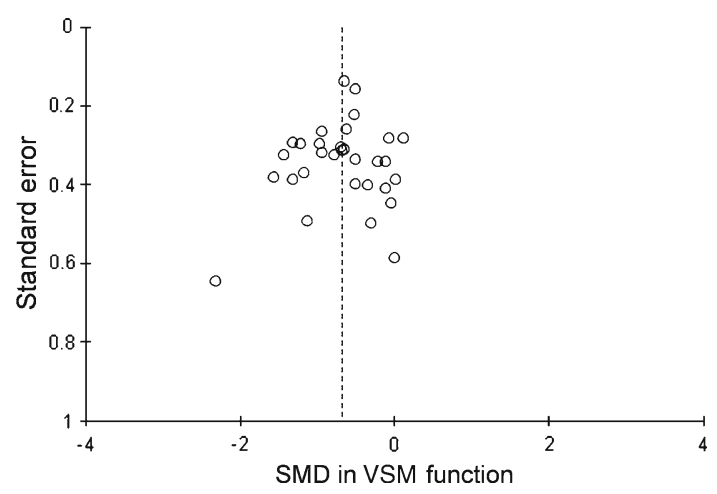

Fig. 4 Funnel plot of studies included in the meta-analysis. Funnel plot asymmetry: $p=0.97$ and $p=0.52$ according to Begg and Mazumdar's rank correlation test and Egger's test, respectively [18]

assessment and prevent cardiovascular complications in individuals with type 2 diabetes.

Another major finding of this meta-analysis was that deterioration of VSM function was more advanced in the microcirculation than in the macrocirculation of diabetic subjects. Moreover, when considering distinct vascular techniques, we detected further decrease in VSM function in studies assessing the microcirculation by the iontophoresis technique, but not by plethysmography, compared with those assessing it by NMD in the macrocirculation. This result could be explained by the fact that forearm plethysmography, besides assessing the microcirculation (vessels $<150 \mu \mathrm{m}$ in diameter, primarily assessed by iontophoresis), also evaluates in part the response of larger vessels such as small resistance arteries. Interestingly, experimental studies reported that induced insulin resistance blocked VSM cell activation in the microcirculation without altering macrovascular blood flow in mice [52]. In humans, locally administered insulin causes a specific increase in VSM activity in the microcirculation of healthy adults [53]. Hence, VSM cells in the pre-capillary arterioles are proposed to be the primary vascular target for insulin, leading to a higher capillary recruitment, and thus play a decisive role in the availability of insulin and glucose to body tissues [54]. Accordingly, the aggravation of VSM dysfunction recognised in the microcirculation of individuals with type 2 diabetes may be a distinctive feature of this metabolic disorder associated with vascular insulin resistance.

VSM function was inversely related with traditional cardiovascular risk factors such as arterial pressure, BMI and triacylglycerols. These results are consistent with the previously reported association between hypertension and VSM dysfunction in individuals with type 2 diabetes [35, 50, 55]. A novel finding arising from this meta-analysis is that both BMI and triacylglycerols were related to VSM dysfunction in the microcirculation, but not in the macrocirculation, of diabetic subjects. Thus, the well-known adverse impact of adiposity and elevated triacylglycerol levels on vascular function might be crucial in the microcirculation of individuals with type 2 diabetes, as recently suggested [56]. Likewise, HDL-cholesterol did not predict VSM function in the macrocirculation, while it showed a strong positive association with VSM function in the microcirculation of diabetic subjects. Consequently, the specific influence of HDLcholesterol in the microcirculation could explain, in part, its prognostic value in type 2 diabetes [57, 58]. In vitro studies indicate that HDL-cholesterol may have numerous direct actions in VSM cells [59]. One such action is the enhancement of VSM relaxation through the upregulation of cyclooxygenase type 2 expression and increased prostacyclin synthesis [60]. Additionally, sphingosine 1-phosphate, a lysosphingolipid component of HDL-cholesterol [61], might play a significant role in preserving nitric oxide bioavailability by inhibiting the generation of reactive oxygen species in VSM cells [62], thence favouring VSM function. Otherwise, the reasons underlying the lack of significant associations between BMI, triacylglycerols and HDL-cholesterol and VSM function in the macrocirculation of individuals with type 2 diabetes are unclear and need further investigation. Taking together, traditional risk factors have an adverse impact on VSM function and explain, to some extent, the aforementioned aggravation of VSM dysfunction in the microcirculation of individuals with type 2 diabetes.

Unexpectedly, VSM function was not related to markers of glucose homeostasis. This result may be partially explained by the effects of oral hypoglycaemic agents and insulin treatments (Table 1). Moreover, fewer studies reported markers of glucose homeostasis than other variable risk factors studied in this meta-analysis (Table 2), meaning that these markers possessed a reduced statistical power. On the other hand, although use of antihypertensive and/or lipidlowering drugs in diabetic subjects was also reported $[23,25$, $26,31,35,37,43,48]$, most studies included in the metaanalyses either excluded those subjects $[22,33,34,36,42$, 45-47] or interrupted treatment before examination [31, 35, 37, 43]. This may account for the detected associations between VSM function and risk factors related to arterial pressure and dyslipidaemia (Fig. 3).

There are some limitations and strengths in the present meta-analysis. Significant heterogeneity was observed among the enrolled studies. However, a comprehensive evaluation of the sources of heterogeneity was performed through subgroup analyses. Additionally, considering that our primary outcome was an SMD in VSM function between diabetic and control subjects, we use the difference in study variables between diabetic and control subjects instead of baseline value of diabetic study variables, to search for moderating factors with accuracy. We also noted that estimates of statistical heterogeneity were moderate by current convention [63]. Individual patient data were not needed in our analysis, since the required aggregate data and standard 
errors could be fully obtained from the published articles themselves [64]. Nonetheless, some potentially relevant studies were excluded from the analysis because control subjects were not age-matched with diabetic subjects. Finally, the quality of studies was evaluated by specific tools for the quality assessment of observational research $[14,15]$. A low bias risk was estimated for study quality, and no risk of publication and reporting biases was detected.

In conclusion, this meta-analysis demonstrated the impairment of VSM function in individuals with type 2 diabetes. Therefore, in addition to the endothelium, the VSM needs to be considered as a potential cause of vascular dysfunction in type 2 diabetes. Moreover, individuals with type 2 diabetes exhibited an aggravation of VSM dysfunction in the microcirculation compared with the macrocirculation. This may be a distinctive feature in type 2 diabetes, associated with vascular insulin resistance, reported previously only in animals. We believe that the present study provides further evidence that risk factors such as adiposity, arterial pressure, triacylglycerols and especially HDL-cholesterol should be tightly controlled in individuals with type 2 diabetes in order to reduce the cardiovascular risk in this population.

Funding DM is supported by a grant from the French Society of Vascular Medicine 2010-2012. ER is supported by a PROMETEO/ 2012/007 Award from Generalitat Valenciana and a grant from Instituto de Salud Carlos III-FEDER (PS09/01093).

Duality of interest The authors declare that there is no duality of interest associated with this manuscript.

Contribution statement DM was responsible for the concept and design of the study. Acquisition of data was carried out by DM and AV. DM, GW, APM, NVS, ER and AV analysed and interpreted the data. Drafting of the manuscript was carried out by DM. DM, GW, APM, NVS, ER and AV critically revised the manuscript for important intellectual content. Statistical expertise was provided by DM and administrative, technical or material support was provided by DM, NVS and ER. All authors approved the final version of the manuscript.

DM had full access to all the data in the study and takes responsibility for the integrity of the data and the accuracy of the data analysis.

\section{References}

1. Zimmet P, Alberti KG, Shaw J (2001) Global and societal implications of the diabetes epidemic. Nature 414:782-787

2. Engelgau MM, Geiss LS, Saaddine JB et al (2004) The evolving diabetes burden in the United States. Ann Intern Med 140:945-950

3. Scholte AJ, Schuijf JD, Kharagjitsingh AV et al (2008) Prevalence of coronary artery disease and plaque morphology assessed by multi-slice computed tomography coronary angiography and calcium scoring in asymptomatic patients with type 2 diabetes. Heart 94:290-295

4. Corretti MC, Anderson TJ, Benjamin EJ et al (2002) Guidelines for the ultrasound assessment of endothelial-dependent flow-mediated vasodilation of the brachial artery: a report of the International
Brachial Artery Reactivity Task Force. J Am Coll Cardiol 39:257-265

5. Morris SJ, Shore AC (1996) Skin blood flow responses to the iontophoresis of acetylcholine and sodium nitroprusside in man: possible mechanisms. J Physiol 496:531-542

6. Ignarro LJ, Lippton H, Edwards JC et al (1981) Mechanism of vascular smooth muscle relaxation by organic nitrates, nitrites, nitroprusside and nitric oxide: evidence for the involvement of Snitrosothiols as active intermediates. J Pharmacol Exp Ther 218:739-749

7. Schachinger V, Britten MB, Zeiher AM (2000) Prognostic impact of coronary vasodilator dysfunction on adverse long-term outcome of coronary heart disease. Circulation 101:1899-1906

8. Gokce N, Keaney JF Jr, Hunter LM et al (2003) Predictive value of noninvasively determined endothelial dysfunction for long-term cardiovascular events in patients with peripheral vascular disease. J Am Coll Cardiol 41:1769-1775

9. Kullo IJ, Malik AR, Bielak LF, Sheedy PF 2nd, Turner ST, Peyser PA (2007) Brachial artery diameter and vasodilator response to nitroglycerine, but not flow-mediated dilatation, are associated with the presence and quantity of coronary artery calcium in asymptomatic adults. Clin Sci 112:175-182

10. Celermajer DS, Sorensen KE, Gooch VM et al (1992) Noninvasive detection of endothelial dysfunction in children and adults at risk of atherosclerosis. Lancet 340:1111-1115

11. Hadi HA, Suwaidi JA (2007) Endothelial dysfunction in diabetes mellitus. Vasc Health Risk Manag 3:853-876

12. Vinet A, Karpoff L, Walther G et al (2011) Vascular reactivity at rest and during exercise in middle-aged obese men: effects of shortterm, low-intensity, exercise training. Int J Obes 35:820-828

13. Stroup DF, Berlin JA, Morton SC et al (2000) Meta-analysis of observational studies in epidemiology: a proposal for reporting. Meta-analysis Of Observational Studies in Epidemiology (MOOSE) group. JAMA 283:2008-2012

14. Ross LE, Grigoriadis S, Mamisashvili L et al (2011) Quality assessment of observational studies in psychiatry: an example from perinatal psychiatric research. Int J Methods Psychiatr Res 20:224-234

15. Sprung VS, Atkinson G, Cuthbertson DJ et al (2013) Endothelial function measured using flow-mediated dilation in polycystic ovary syndrome: a meta-analysis of the observational studies. Clin Endocrinol 78:438-446

16. Cohen J (1988) Statistical power analysis for the behavioral sciences, 2nd edn. Lawrence Erlbaum Associates Publishers, Hillsdale

17. DerSimonian R, Laird N (1986) Meta-analysis in clinical trials. Control Clin Trials 7:177-188

18. Egger M, Davey Smith G, Schneider M, Minder C (1997) Bias in meta-analysis detected by a simple, graphical test. BMJ 315:629634

19. Chittari MV, McTernan P, Bawazeer N et al (2011) Impact of acute hyperglycaemia on endothelial function and retinal vascular reactivity in patients with Type 2 diabetes. Diabet Med 28:450-454

20. Natali A, Toschi E, Baldeweg S et al (2005) Haematocrit, type 2 diabetes, and endothelium-dependent vasodilatation of resistance vessels. Eur Heart J 26:464-471

21. Watts GF, O'Brien SF, Silvester W, Millar JA (1996) Impaired endothelium-dependent and independent dilatation of forearm resistance arteries in men with diet-treated non-insulin-dependent diabetes: role of dyslipidaemia. Clin Sci 91:567-573

22. Anderson RA, Evans ML, Ellis GR et al (2001) The relationships between post-prandial lipaemia, endothelial function and oxidative stress in healthy individuals and patients with type 2 diabetes. Atherosclerosis 154:475-483

23. Beer S, Feihl F, Ruiz J et al (2008) Comparison of skin microvascular reactivity with hemostatic markers of endothelial dysfunction and damage in type 2 diabetes. Vasc Health Risk Manag 4:14491458 
24. Brooks BA, Franjic B, Ban CR et al (2008) Diastolic dysfunction and abnormalities of the microcirculation in type 2 diabetes. Diabetes Obes Metab 10:739-746

25. Bruno RM, Penno G, Daniele G et al (2012) Type 2 diabetes mellitus worsens arterial stiffness in hypertensive patients through endothelial dysfunction. Diabetologia 55:1847-1855

26. Cipolla MJ, Harker CT, Porter JM (1996) Endothelial function and adrenergic reactivity in human type-II diabetic resistance arteries. J Vasc Surg 23:940-949

27. Enderle MD, Benda N, Schmuelling RM, Haering HU, Pfohl M (1998) Preserved endothelial function in IDDM patients, but not in NIDDM patients, compared with healthy subjects. Diabetes Care 21:271-277

28. Heitzer T, Finckh B, Albers S, Krohn K, Kohlschutter A, Meinertz $\mathrm{T}$ (2001) Beneficial effects of alpha-lipoic acid and ascorbic acid on endothelium-dependent, nitric oxide-mediated vasodilation in diabetic patients: relation to parameters of oxidative stress. Free Radic Biol Med 31:53-61

29. Ifrim S, Vasilescu R (2004) Early detection of atherosclerosis in type 2 diabetic patients by endothelial dysfunction and intimamedia thickness. Rom J Intern Med 42:343-354

30. Ihlemann N, Stokholm KH, Eskildsen PC (2002) Impaired vascular reactivity is present despite normal levels of von Willebrand factor in patients with uncomplicated Type 2 diabetes. Diabet Med 19:476-481

31. Karabag T, Kaya A, Temizhan A, Koc F, Yavuz S, Cam S (2007) The influence of homocysteine levels on endothelial function and their relation with microvascular complications in T2DM patients without macrovascular disease. Acta Diabetol 44:69-75

32. Kimura Y, Matsumoto M, Miyauchi E, Deng YB, Iwai K, Hattori H (2001) Noninvasive detection of endothelial dysfunction in elderly with NIDDM by ultrasonography. Echocardiography 18:559-564

33. Lim SC, Caballero AE, Arora S et al (1999) The effect of hormonal replacement therapy on the vascular reactivity and endothelial function of healthy individuals and individuals with type 2 diabetes. $\mathrm{J}$ Clin Endocrinol Metab 84:4159-4164

34. Lim SC, Caballero AE, Smakowski P, LoGerfo FW, Horton ES, Veves A (1999) Soluble intercellular adhesion molecule, vascular cell adhesion molecule, and impaired microvascular reactivity are early markers of vasculopathy in type 2 diabetic individuals without microalbuminuria. Diabetes Care 22:1865-1870

35. Ma L, Zhao S, Li J, Zhou Q, Gao M (2001) Interaction of hypertension and diabetes on impairment of endothelial function. Chin Med J (Engl) 114:563-567

36. Makimattila S, Liu ML, Vakkilainen J et al (1999) Impaired endothelium-dependent vasodilation in type 2 diabetes. Relation to LDL size, oxidized LDL, and antioxidants. Diabetes Care 22:973-981

37. Matsumoto N, Ishimura E, Taniwaki $\mathrm{H}$ et al (2002) Smoking and proteinuria impair vasodilatory response of intrarenal arteries to nitroglycerine in patients with type 2 diabetes mellitus. Nephrol Dial Transplant 17:608-613

38. Pitei DL, Watkins PJ, Edmonds ME (1997) NO-dependent smooth muscle vasodilatation is reduced in NIDDM patients with peripheral sensory neuropathy. Diabet Med 14:284-290

39. Sivitz WI, Wayson SM, Bayless ML, Sinkey CA, Haynes WG (2007) Obesity impairs vascular relaxation in human subjects: hyperglycemia exaggerates adrenergic vasoconstriction arterial dysfunction in obesity and diabetes. J Diabetes Complications 21:149-157

40. Sokolnicki LA, Roberts SK, Wilkins BW, Basu A, Charkoudian N (2007) Contribution of nitric oxide to cutaneous microvascular dilation in individuals with type 2 diabetes mellitus. Am J Physiol Endocrinol Metab 292:E314-E318

41. Tan KC, Chow WS, Ai VH, Metz C, Bucala R, Lam KS (2002) Advanced glycation end products and endothelial dysfunction in type 2 diabetes. Diabetes Care 25:1055-1059
42. van de Ree MA, Huisman MV, de Man FH, van der Vijver JC, Meinders AE, Blauw GJ (2001) Impaired endothelium-dependent vasodilation in type 2 diabetes mellitus and the lack of effect of simvastatin. Cardiovasc Res 52:299-305

43. van Etten RW, de Koning EJ, Verhaar MC, Gaillard CA, Rabelink TJ (2002) Impaired NO-dependent vasodilation in patients with Type II (non-insulin-dependent) diabetes mellitus is restored by acute administration of folate. Diabetologia 45:1004-1010

44. Vehkavaara S, Yki-Jarvinen H (2004) 3.5 years of insulin therapy with insulin glargine improves in vivo endothelial function in type 2 diabetes. Arterioscler Thromb Vasc Biol 24:325-330

45. Williams SB, Cusco JA, Roddy MA, Johnstone MT, Creager MA (1996) Impaired nitric oxide-mediated vasodilation in patients with non-insulin-dependent diabetes mellitus. J Am Coll Cardiol 27:567-574

46. Woodman RJ, Playford DA, Watts GF (2006) Basal production of nitric oxide (NO) and non-NO vasodilators in the forearm microcirculation in Type 2 diabetes: associations with blood pressure and HDL cholesterol. Diabetes Res Clin Pract 71:59-67

47. Woodman RJ, Watts GF, Playford DA, Best JD, Chan DC (2005) Oxidized LDL and small LDL particle size are independently predictive of a selective defect in microcirculatory endothelial function in type 2 diabetes. Diabetes Obes Metab 7:612-617

48. Woodman RJ, Watts GF, Puddey IB et al (2002) Leukocyte count and vascular function in Type 2 diabetic subjects with treated hypertension. Atherosclerosis 163:175-181

49. Adams MR, Robinson J, McCredie R et al (1998) Smooth muscle dysfunction occurs independently of impaired endotheliumdependent dilation in adults at risk of atherosclerosis. J Am Coll Cardiol 32:123-127

50. Akamatsu D, Sato A, Goto H et al (2010) Nitroglycerin-mediated vasodilatation of the brachial artery may predict long-term cardiovascular events irrespective of the presence of atherosclerotic disease. J Atheroscler Thromb 17:1266-1274

51. Kawano N, Emoto M, Mori K et al (2012) Association of endothelial and vascular smooth muscle dysfunction with cardiovascular risk factors, vascular complications, and subclinical carotid atherosclerosis in type 2 diabetic patients. J Atheroscler Thromb 19:276-284

52. Newman JM, Dwyer RM, St-Pierre P, Richards SM, Clark MG, Rattigan S (2009) Decreased microvascular vasomotion and myogenic response in rat skeletal muscle in association with acute insulin resistance. J Physiol 587:2579-2588

53. Rossi M, Maurizio S, Carpi A (2005) Skin blood flowmotion response to insulin iontophoresis in normal subjects. Microvasc Res 70:17-22

54. Clark MG (2008) Impaired microvascular perfusion: a consequence of vascular dysfunction and a potential cause of insulin resistance in muscle. Am J Physiol Endocrinol Metab 295:E732-E750

55. Naka KK, Papathanassiou K, Bechlioulis A et al (2012) Determinants of vascular function in patients with type 2 diabetes. Cardiovasc Diabetol 11:127

56. Wiernsperger N, Rapin JR (2012) Microvascular diseases: is a new era coming? Cardiovasc Hematol Agents Med Chem 10:167-183

57. Kannel WB (1990) CHD risk factors: a Framingham study update. Hosp Pract (Off Ed) 25:119-127, 130

58. Nichols GA, Vupputuri S, Rosales AG (2011) Change in highdensity lipoprotein cholesterol and risk of subsequent hospitalization for coronary artery disease or stroke among patients with type 2 diabetes mellitus. Am J Cardiol 108:1124-1128

59. Mineo C, Shaul PW (2012) Novel biological functions of highdensity lipoprotein cholesterol. Circ Res 111:1079-1090

60. Vinals M, Martinez-Gonzalez J, Badimon JJ, Badimon L (1997) HDL-induced prostacyclin release in smooth muscle cells is 
dependent on cyclooxygenase-2 (Cox-2). Arterioscler Thromb Vasc Biol 17:3481-3488

61. Argraves KM, Argraves WS (2007) HDL serves as a S1P signaling platform mediating a multitude of cardiovascular effects. J Lipid Res 48:2325-2333

62. Tolle M, Pawlak A, Schuchardt M et al (2008) HDL-associated lysosphingolipids inhibit $\mathrm{NAD}(\mathrm{P}) \mathrm{H}$ oxidase-dependent monocyte chemoattractant protein-1 production. Arterioscler Thromb Vasc Biol 28:1542-1548

63. Higgins JP, Thompson SG, Deeks JJ, Altman DG (2003) Measuring inconsistency in meta-analyses. BMJ 327:557-560

64. Riley RD, Lambert PC, Abo-Zaid G (2010) Meta-analysis of individual participant data: rationale, conduct, and reporting. BMJ 340:c221 